\title{
Laser Imaging Facilitates Early Detection of Synchronous Adenocarcinomas in Patients with Barrett's Esophagus
}

\author{
Chihiro Iwashita ${ }^{1}$, Yoshimasa Miura ${ }^{1}$, Hiroyuki Osawa ${ }^{1}$, Takahito Takezawa ${ }^{1}$, Yuji Ino ${ }^{1}$, Masahiro 0kada ${ }^{1}$, Alan K. Lefor ${ }^{2}$ and \\ Hironori Yamamoto ${ }^{1}$ \\ ${ }^{1}$ Division of Gastroenterology, Department of Internal Medicine, ${ }^{2}$ Department of Surgery, Jichi Medical University, Shimotsuke, Japan
}

Barrett's adenocarcinoma may occur in multiple sites, and recurrence and metachronous lesions are the major problems with endoscopic resection. Therefore, early detection of such lesions is ideal to achieve complete resection and obtain improved survival rates with minimally invasive treatment. Laser imaging systems allow multiple modalities of endoscopic imaging by using white light laser, flexible spectral imaging color enhancement (FICE), blue laser imaging (BLI), and linked color imaging even at a distant view. However, the usefulness of these modalities has not been sufficiently reported regarding Barrett's adenocarcinoma. Here, we report on a patient with three synchronous lesions followed by one metachronous lesion in a long segment with changes of Barrett's esophagus, all diagnosed with this new laser endoscopic imaging system and enhanced by using FICE and/or BLI with high contrast compared with the surrounding mucosa. Laser endoscopic imaging may facilitate the detection of malignancies in patients with early Barrett's adenocarcinoma. Clin Endosc 2017;50:81-86

Key Words: Barrett esophagus; Early synchronous neoplasms; Endoscopic submucosal dissection; Blue laser imaging; Flexible spectral image color enhancement

\section{INTRODUCTION}

Barrett's esophagus is the main risk factor for the development of esophageal adenocarcinoma. Currently, the most widely used endoscopic therapies for Barrett's adenocarcinoma include endoscopic mucosal resection and endoscopic submucosal dissection (ESD), ${ }^{1,2}$ which result in complete resection. However, several investigators have reported on the occurrence of metachronous recurrence after the initial endoscopic resection. ${ }^{3-5}$ This may occur as multiple lesions at the initial endoscopic examination, and therefore early detection of such lesions is ideal to achieve complete resection and

Received: January 20, 2016 Revised: April 20, 2016

Accepted: April 20, 2016

Correspondence: Hiroyuki Osawa

Division of Gastroenterology, Department of Internal Medicine, Jichi Medical University, 3311-1 Yakushiji, Shimotsuke 329-0498, Japan

Tel: +81-285-58-7348, Fax: +81-285-44-8297, E-mail: osawa@jichi.ac.jp

(cc) This is an Open Access article distributed under the terms of the Creative Commons Attribution Non-Commercial License (http://creativecommons.org/ licenses/by-nc/3.0) which permits unrestricted non-commercial use, distribution, and reproduction in any medium, provided the original work is properly cited. obtain improved survival rates with minimally invasive treatment. $^{4-6}$

Narrow band imaging (NBI) is useful for the detailed examination of adenocarcinoma associated with Barrett's esophagus, which includes detection of the irregular microvessels of esophageal and gastric cancer at high magnification. ${ }^{4-6}$ However, this technique has limitations, such as the difficulty to brightly view distant lesions in a large luminal organ such as the stomach.

Recently, a laser endoscopic imaging system (Fujifilm Co., Tokyo, Japan) has been developed to facilitate new diagnostic approaches for gastrointestinal lesions. ${ }^{7,8}$ This system allows multiple modalities of endoscopic imaging by using white light laser, flexible spectral imaging color enhancement (FICE), blue laser imaging (BLI), and linked color imaging even at a distant view. Therefore, this system may be useful for the detection of adenocarcinoma associated with Barrett's esophagus. We report on a patient with three synchronous lesions followed by one metachronous lesion in a long segment with changes of Barrett's esophagus, all diagnosed with this new laser endoscopic imaging system. 


\section{Ce cuncen Enooscopr}
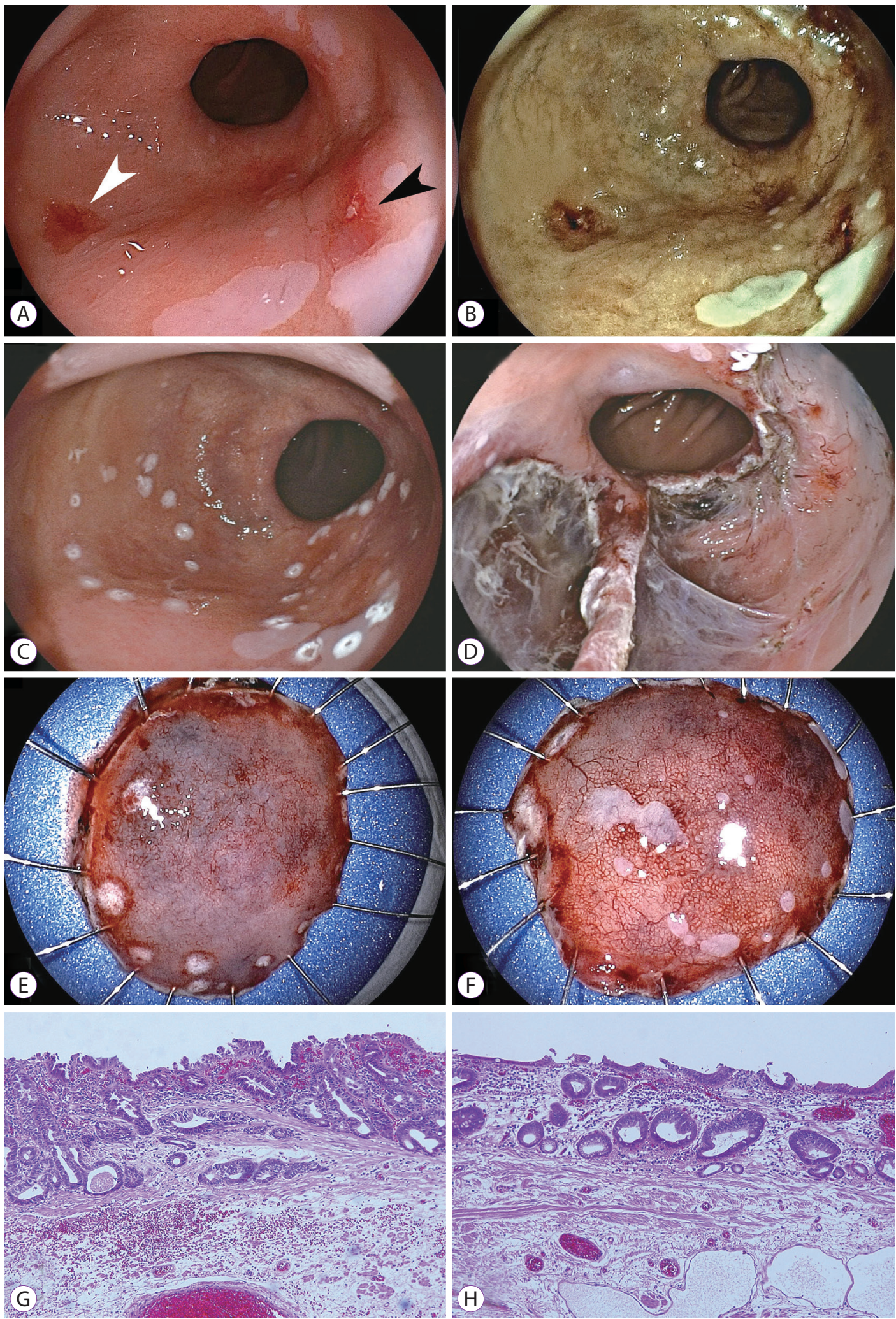

Fig. 1. (A) Two reddish lesions (white and black arrowheads) are seen in the distal esophagus. (B) Flexible spectral imaging color enhancement shows both lesions that feature depressed-type cancers. The most distal lesion shows inflammatory changes. (C) Markings are placed $5 \mathrm{~mm}$ outside each of the two cancers. (D) Malignant lesions were resected with endoscopic submucosal dissection (ESD). (E) Specimens resected from the anterior wall by using ESD are shown. (F) Similarly, specimens from the posterior wall resected with ESD are shown. $(\mathrm{G})$ Resected specimens from the anterior wall show well-differentiated adenocarcinoma (H\&E stain, $\times 100)$. (H) Similarly, specimens from the posterior wall show well-differentiated adenocarcinoma (H\&E stain, $\times 100$ ). 


\section{CASE REPORT}

A 71-year-old woman was referred for further evaluation of an uneven surface mucosa in the esophagus, in a Barrett's area on the anterior wall of the distal esophagus where well-differentiated adenocarcinoma was diagnosed with biopsy. The patient has a history of cerebral infarction at age 50 years, from which she recovered, except for moderate left hemiparesis. Although she also has a history of chronic heart failure, her cardiac function was improved. Her physical examination was normal; blood and serum chemistry tests including coagula- tion studies were normal; and chest and abdominal computed tomography scans showed no metastatic lesions. She has been routinely followed up for the development of malignant lesions in a long segment of Barrett's esophagus.

According to the Prague classification, the circumferential extent $(\mathrm{C})$ of Barrett's esophagus was $4 \mathrm{~cm}$ and the maximum length (M) was $7 \mathrm{~cm}$. By using a laser imaging system, we found reddish lesions on the anterior and posterior walls of the distal esophagus in white light laser images (Fig. 1). In addition, another lesion in the middle esophagus was detected as a brownish area, by using FICE and BLI (Fig. 2). All
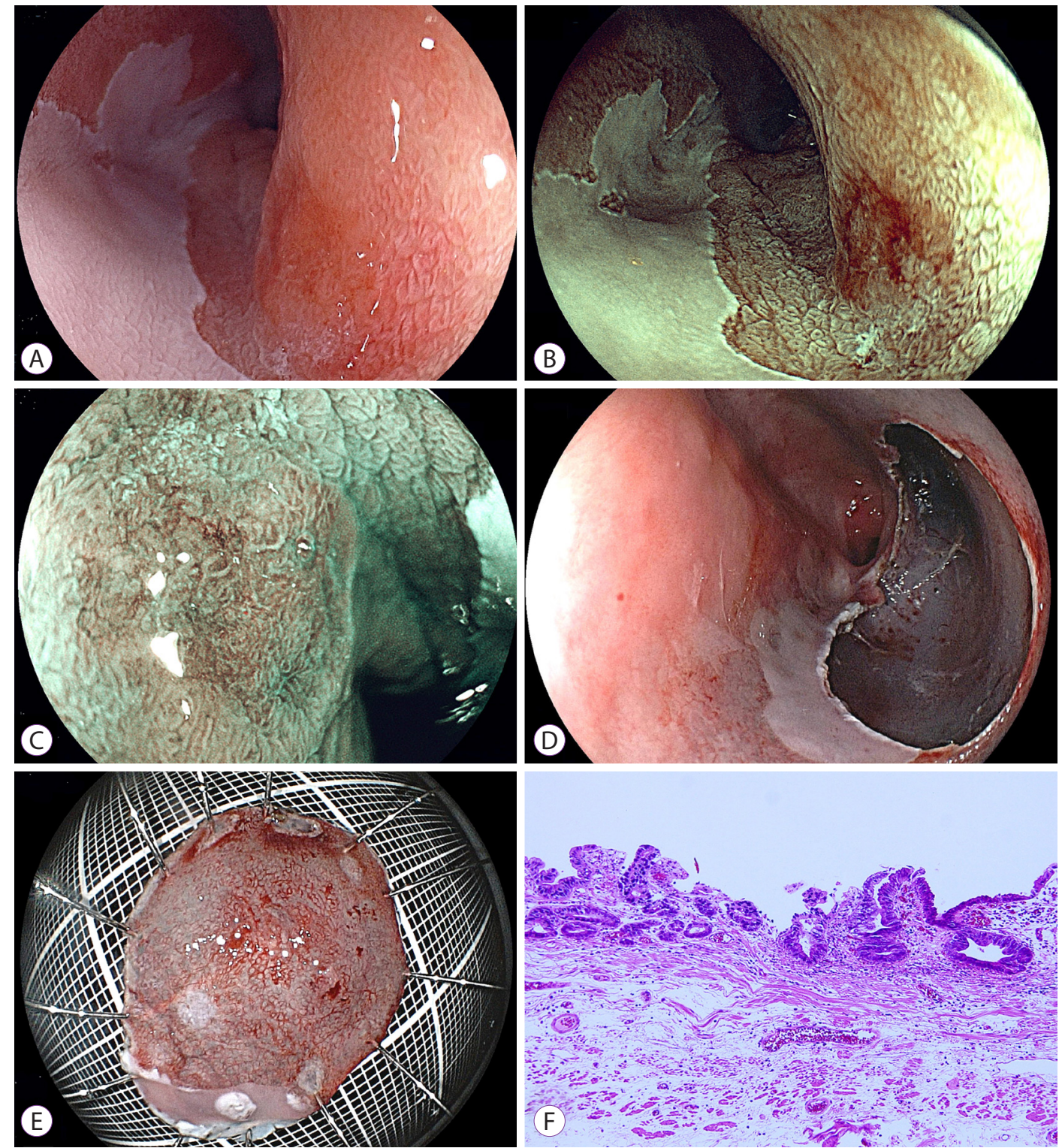

Fig. 2. (A) A slightly reddish lesion is seen in the middle esophagus; however, it is difficult to establish the diagnosis. (B) Flexible spectral imaging color enhancement shows a reddish depressed lesion distinct from the surrounding mucosa. (C) Magnified blue laser imaging shows a brown malignant lesion with an irregular surface pattern distinct from the surrounding mucosa and an irregular vascular pattern in one portion of the tumor. (D) Malignant lesion was resected with endoscopic submucosal dissection (ESD). (E) Specimens resected by using ESD are shown. (F) Pathological evaluation of the resected specimen shows well-differentiated adenocarcinoma (H\&E stain, $\times 100)$. 

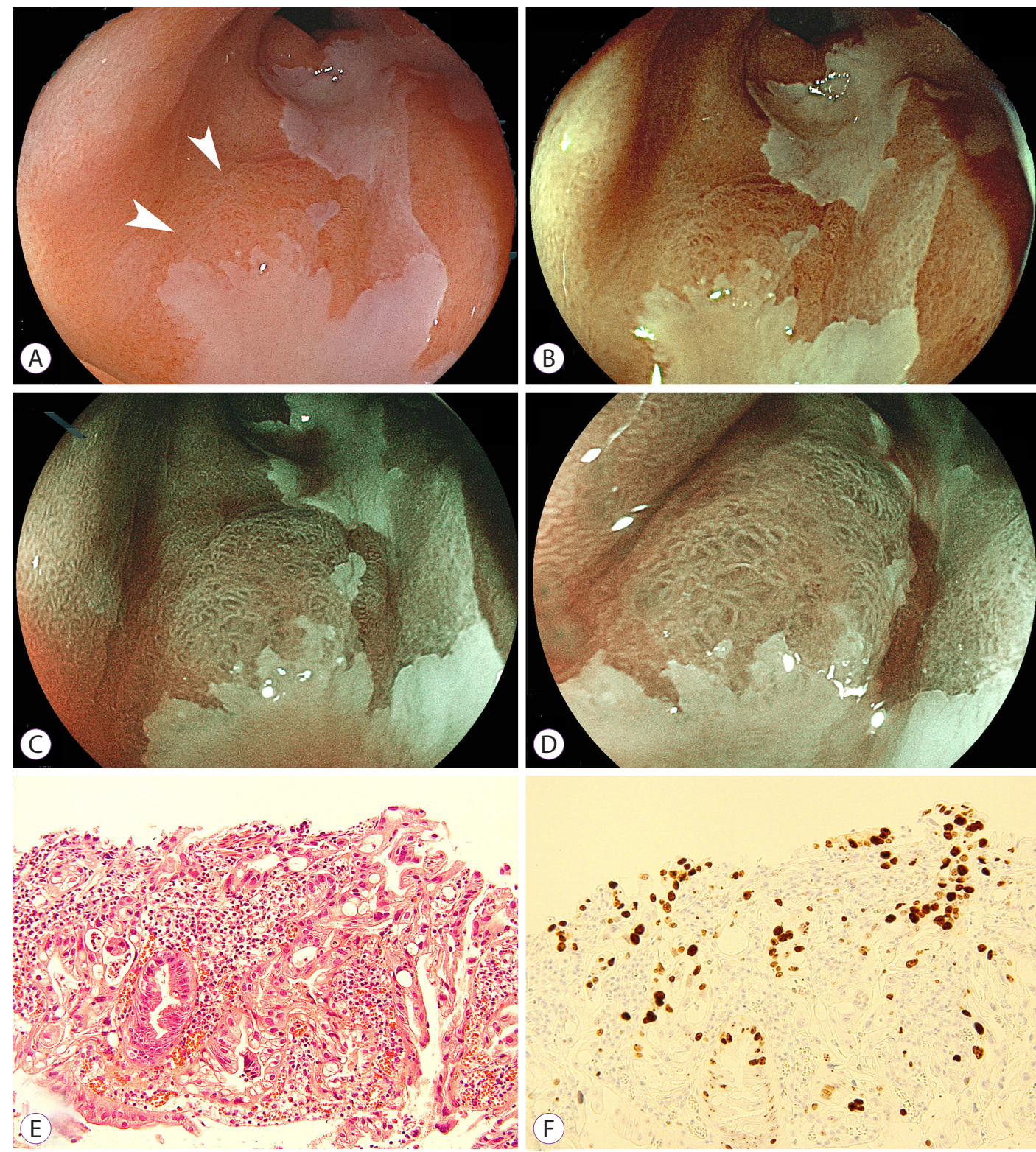

Fig. 3. (A) A slightly elevated lesion with an irregular surface (white arrowheads) is seen in the middle esophagus; however, it is difficult to establish the diagnosis. (B) Flexible spectral imaging color enhancement shows a slightly brown lesion distinct from the surrounding mucosa. (C) Low-magnification blue laser imaging shows an irregular surface pattern of the elevated malignant lesion distinct from the surrounding mucosa. (D) Half-magnification blue laser imaging more clearly shows the irregular surface pattern of a malignant lesion. (E) Biopsy specimen shows atypical glands suggesting cancer (H\&E stain, $\times 100)$. (F) Immunohistochemical staining reveals Ki-67-positive cells mainly confined to the surface layer of the biopsy specimen, suggesting a malignant lesion (H\&E stain, $\times 100$ ).

three lesions were 0-IIc early cancers with slight reddish color changes in the laser white light images: two lesions measuring 15 and $10 \mathrm{~mm}$ in diameter on the anterior and posterior walls of the distal esophagus, respectively, and another lesion measuring $5 \mathrm{~mm}$ in the middle esophagus. FICE and BLI enhanced all three lesions with high contrast, compared with the surrounding mucosa. Magnification BLI images revealed an irregular surface pattern on the brown lesion compared with the surrounding mucosa and an irregular vascular pattern in one portion of the tumor. All biopsy specimens showed atypi- cal cells, suspicious for well-differentiated adenocarcinoma.

Three synchronous early adenocarcinomas in a long segment of Barrett's esophagus were diagnosed. ESD was scheduled for the two lesions in the distal esophagus at the first resection and for the lesion in the middle esophagus 3 months later.

All lesions were resected completely through ESD with sodium hyaluronate solution without difficulties. The en bloc resected mucosa measured $27 \mathrm{~mm}$ for the anterior wall lesion in the distal esophagus, $26 \mathrm{~mm}$ for the posterior wall lesion, and 
$25 \mathrm{~mm}$ for the middle esophageal lesion. The tumors were 10, 5 , and $13 \mathrm{~mm}$, respectively.

Pathological diagnosis of the resected specimens showed well-differentiated adenocarcinoma in all lesions (Figs. 1G, H, $2 \mathrm{~F})$. All tumors were located within the lamina propria mucosa with tumor-free vertical and horizontal margins. Double muscularis mucosae were seen in the area beneath the malignant cells in the resected specimens from the anterior wall of the lower esophagus and the middle esophagus. No tumor cells invaded the deep muscularis mucosae. Esophageal glands were found in the submucosa of the posterior wall of the lower esophagus. Lymphovascular invasion was not seen in the three resected specimens. These findings are all characteristic of adenocarcinoma in Barrett's esophagus.

The patient was followed up with endoscopy with laser imaging every 6 months, and a fourth lesion was detected 2 years after the final ESD procedure. This new lesion had a slightly reddish area on white light laser images and an irregular surface pattern on BLI bright images (Fig. 3). Histopathology revealed well-differentiated adenocarcinoma. However, this lesion was not resected because the patient's cardiac function had worsened.

\section{DISCUSSION}

This report demonstrates the early detection of three synchronous and one metachronous neoplasms in a patient with a long segment of Barrett's esophagus, by using white light, FICE, and BLI images produced with a newly introduced laser imaging system. Three synchronous malignancies were completely resected with ESD.

Patients with a diagnosis of Barrett's esophagus are at a risk of developing high-grade dysplasia or esophageal adenocarcinoma. Recurrence and metachronous lesions have been shown to be the major problems with endoscopic resection in patients with early Barrett's neoplasia. ${ }^{3-5}$ The prevalence of metachronous lesions or disease recurrence increases to $14 \%$ within 12 months, and to $21.5 \%$ at 5 years. ${ }^{3}$ However, such lesions may be missed at the initial endoscopic examination. Several investigators recommend that even with high-resolution endoscopy, four-quadrant biopsies are necessary after careful evaluation of Barrett's mucosa to exclude synchronous neoplastic lesions. ${ }^{1,2}$ The biopsy specimens provide histological information on the mucosa with Barrett's changes; however, only a small fraction of the mucosa can be sampled. ${ }^{9,10}$ Lesions harboring dysplasia or carcinoma are easily missed. ${ }^{2}$ The laser imaging system can produce high-resolution images even with white light imaging. Before obtaining biopsies, detailed observation by using laser imaging may also be useful for the detection of synchronous neoplasms and may compensate for the disadvantages associated with four-quadrant biopsies.

In the current patient, one missed lesion was detected by using high-resolution white light laser images. Laser endoscopic images are strongly associated with the higher emission intensity of short wavelengths illuminated by using blue laser light rather than xenon light. One of the main advantages is that the higher emission intensity of short wavelengths enables the observation of bright high-resolution images of the mucosal surface at a relatively distant view. Another missed lesion was detected as a brownish area, by using FICE and BLI. BLI and FICE images reveal malignancies with high contrast compared with the surrounding mucosa. Magnifying BLI is closely related to short wavelengths and reveals a clearly irregular microstructural pattern (Figs. $2,3)$. These findings are not seen in the surrounding mucosa. It is noteworthy that these abnormal findings are obtained at relatively low magnification. However, FICE is less related to short wavelengths and cannot sufficiently demonstrate the microstructural pattern, which may be a disadvantage.

In contrast, images of the microvasculature need higher resolution than those of the microstructural pattern and a nearer view with high magnification for a more precise evaluation, even with the laser imaging system. Laser imaging is produced by using longer wavelengths unrelated to absorption by hemoglobin in addition to short wavelengths related to hemoglobin absorption. Longer wavelengths responsible for white light images are associated with imaging of the microstructure with low magnification or at a distant view, but not with the microvasculature on the tumor surface. Thus, laser imaging with low magnification can show the microstructure but does not sufficiently image the microvessels on the tumor surface.

Image-enhanced endoscopy with high magnification allows the detailed observation of the microvascular and microstructural patterns of Barrett's cancer. Several studies with NBI described irregular microvascular and microstructural patterns that were highly associated with high-grade dysplasia/early cancer and showed high sensitivity and specificity of NBI in detecting neoplasia. ${ }^{11-14}$ Singh et al. ${ }^{15}$ reported that Barrett's esophagus had four morphologic patterns. They described that a distorted pit with irregular microvasculature corresponds to malignant lesions. These findings are important in diagnosing Barrett's cancer by using image-enhanced endoscopy such as NBI and BLI.

With the laser imaging system, a recent report revealed that linked color imaging is useful for the early detection of gastric cancer. ${ }^{16}$ In the future, linked color imaging should be evaluated for the detection of Barrett's adenocarcinoma.

In conclusion, laser endoscopic imaging may facilitate the 
detection of malignancies in patients with early Barrett's adenocarcinoma, similar to early gastric cancer. ${ }^{7}$ Further assessment in a large study is necessary to evaluate the usefulness of endoscopic diagnosis for multiple adenocarcinomas in areas of Barrett's esophagus.

\section{Conflicts of Interest}

Hiroyuki Osawa received commercial research funding from FUJIFILM Medical Corporation; Hironori Yamamoto has a consultant relationship with FUJIFILM Corporation, as well as honoraria, grants, and royalties from the company. The other authors have no financial conflicts of interest.

\section{REFERENCES}

1. Bennett C, Vakil N, Bergman J, et al. Consensus statements for management of Barrett's dysplasia and early-stage esophageal adenocarcinoma, based on a Delphi process. Gastroenterology 2012;143:336-346.

2. Gill RS, Singh R. Endoscopic imaging in Barrett's esophagus: current practice and future applications. Ann Gastroenterol 2012;25:89-95.

3. Pech O, Behrens A, May A, et al. Long-term results and risk factor analysis for recurrence after curative endoscopic therapy in 349 patients with high-grade intraepithelial neoplasia and mucosal adenocarcinoma in Barrett's oesophagus. Gut 2008;57:1200-1206.

4. Chennat J, Konda VJ, Ross AS, et al. Complete Barrett's eradication endoscopic mucosal resection: an effective treatment modality for highgrade dysplasia and intramucosal carcinoma. An American single-center experience. Am J Gastroenterol 2009;104:2684-2692.

5. Pech O, May A, Manner H, et al. Long-term efficacy and safety of endoscopic resection for patients with mucosal adenocarcinoma of the esophagus. Gastroenterology 2014;146:652-660.

6. Ikeda $\mathrm{K}$, Isomoto $\mathrm{H}$, Oda $\mathrm{H}$, et al. Endoscopic submucosal dissection of a minute intramucosal adenocarcinoma in Barrett's esophagus. Dig Endosc 2009;21:34-36.

7. Osawa H, Yamamoto $\mathrm{H}$. Present and future status of flexible spectral imaging color enhancement and blue laser imaging technology. Dig Endosc 2014;26 Suppl 1:105-115.

8. Osawa H, Yamamoto H, Miura Y et al. Blue laser imaging provides excellent endoscopic images of upper gastrointestinal lesions. Video J Encycl GI Endosc 2014;1:607-610.

9. Falk GW, Rice TW, Goldblum JR, Richter JE. Jumbo biopsy forceps protocol still misses unsuspected cancer in Barrett's esophagus with high-grade dysplasia. Gastrointest Endosc 1999;49:170-176.

10. Kim SL, Waring JP, Spechler SJ, et al. Diagnostic inconsistencies in Barrett's esophagus. Department of veterans affairs gastroesophageal reflux study group. Gastroenterology 1994;107:945-949.

11. Kara MA, Peters FP, Fockens P, ten Kate FJ, Bergman JJ. Endoscopic video-autofluorescence imaging followed by narrow band imaging for detecting early neoplasia in Barrett's esophagus. Gastrointest Endosc 2006;64:176-185.

12. Sharma P, Bansal A, Mathur S, et al. The utility of a novel narrow band imaging endoscopy system in patients with Barrett's esophagus. Gastrointest Endosc 2006;64:167-175.

13. Anagnostopoulos GK, Yao K, Kaye P, Hawkey CJ, Ragunath K. Novel endoscopic observation in Barrett's oesophagus using high resolution magnification endoscopy and narrow band imaging. Aliment Pharmacol Ther 2007;26:501-507.

14. Curvers WL, Bohmer CJ, Mallant-Hent RC, et al. Mucosal morphology in Barrett's esophagus: interobserver agreement and role of narrow band imaging. Endoscopy 2008;40:799-805.

15. Singh R, Anagnostopoulos GK, Yao K, et al. Narrow-band imaging with magnification in Barrett's esophagus: validation of a simplified grading system of mucosal morphology patterns against histology. Endoscopy 2008;40:457-463.

16. Fukuda H, Miura Y, Hayashi Y, et al. Linked color imaging technology facilitates early detection of flat gastric cancers. Clin J Gastroenterol 2015;8:385-389. 